\title{
Zonificación sísmica del Estado de Tlaxcala, México
}

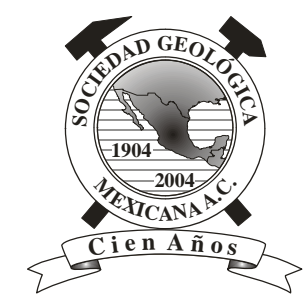

\author{
Javier Lermo-Samaniego*, Isabel Bernal-Esquia \\ Instituto de Ingeniería, Universidad Nacional Autónoma de México, \\ Ciudad Universitaria, 04510 México, D.F. \\ *jles@pumas.iingen.unam.mx
}

\begin{abstract}
Resumen
Para la evaluación y análisis de la sismicidad que se genera en el Estado de Tlaxcala, se han analizado 45 sismos registrados entre 1984 y 2004 (Md<4.0). De esta sismicidad, casi el 70\% está asociada a la actividad del Graben de Puebla, coincidiendo con las trazas del sistema de fallas, así como con la orientación de los mecanismos focales que es principalmente en dirección E-O. Estas características sismotectónicas permitieron realizar una zonificación sísmica, que consta de zonas de alta (I), media (II) y baja (III) sismicidad. La Zona I, la de mayor peligrosidad contiene las ciudades con mayor densidad poblacional del estado: Tlaxcala, Santa Ana Chiautempan, Contla y Apizaco.
\end{abstract}

Palabras clave: zonificación sísmica, Estado de Tlaxcala, sismicidad, mecanismos focales.

Abstract

For the evaluation and analysis of the seismicity that is generated in the State of Tlaxcala, we have analyzed 45 earthquakes that occurred between 1984 and 2004 (Md<4.0). Almost 70\% of such earthquakes is associated with the activity of the Puebla Graben. This agrees with the location of fault systems and oriented focal mechanisms, mainly E-W. These seismotectonic characteristics allowed to establish a seismic zonation, that consists of a high (I), medium (II) and low (III) seismicity zones. Zone I, the one that bears greatest potential seismic hazard contains the cities with greater population density of this state: Tlaxcala, Santa Ana Chiautempan, Contla $y$ Apizaco.

Key words: seismic zonation, State of Tlaxcala, seismicity, focal mechanisms.

\section{Introducción}

El Estado de Tlaxcala, con aproximadamente un millón de habitantes, se ubica en la parte central de la República Mexicana (1944'N a $19^{\circ} 06^{\prime} \mathrm{N}$ y $97^{\circ} 38^{\prime} \mathrm{O}$ a $\left.98^{\circ} 43^{\prime} \mathrm{O}\right)$ dentro de la Faja Volcánica Mexicana (FVM). Este territorio accidentado se caracteriza por la presencia de mesetas, lomeríos, llanuras, un cañón y el volcán La Malinche o Matlacueyetl (4461 m.s.n.m.), como se puede observar en la Figura 1. Asimismo, es importante la presencia de plegamientos que se orientan predominantemente en dirección E-O, dando origen a depresiones, fallas y dislocaciones que en la actualidad están generando sismos de magnitud moderada y pequeña. En la Figura 1, también se muestra los principales sistemas de fallas que existen en esta región, como son las Fallas Tlaxcala, Zacatelco, Huejotzingo y Tepeaca que se localizan, las dos primeras al centro y sur del estado de Tlaxcala, respectivamente; mientras que las otras dos en el estado de Puebla, respectivamente. Las cuatro fallas son de tipo normal y están orientadas en dirección E-O, sin embargo las dos primeras, presentan buzamiento hacia el sur y las otras dos hacia el 


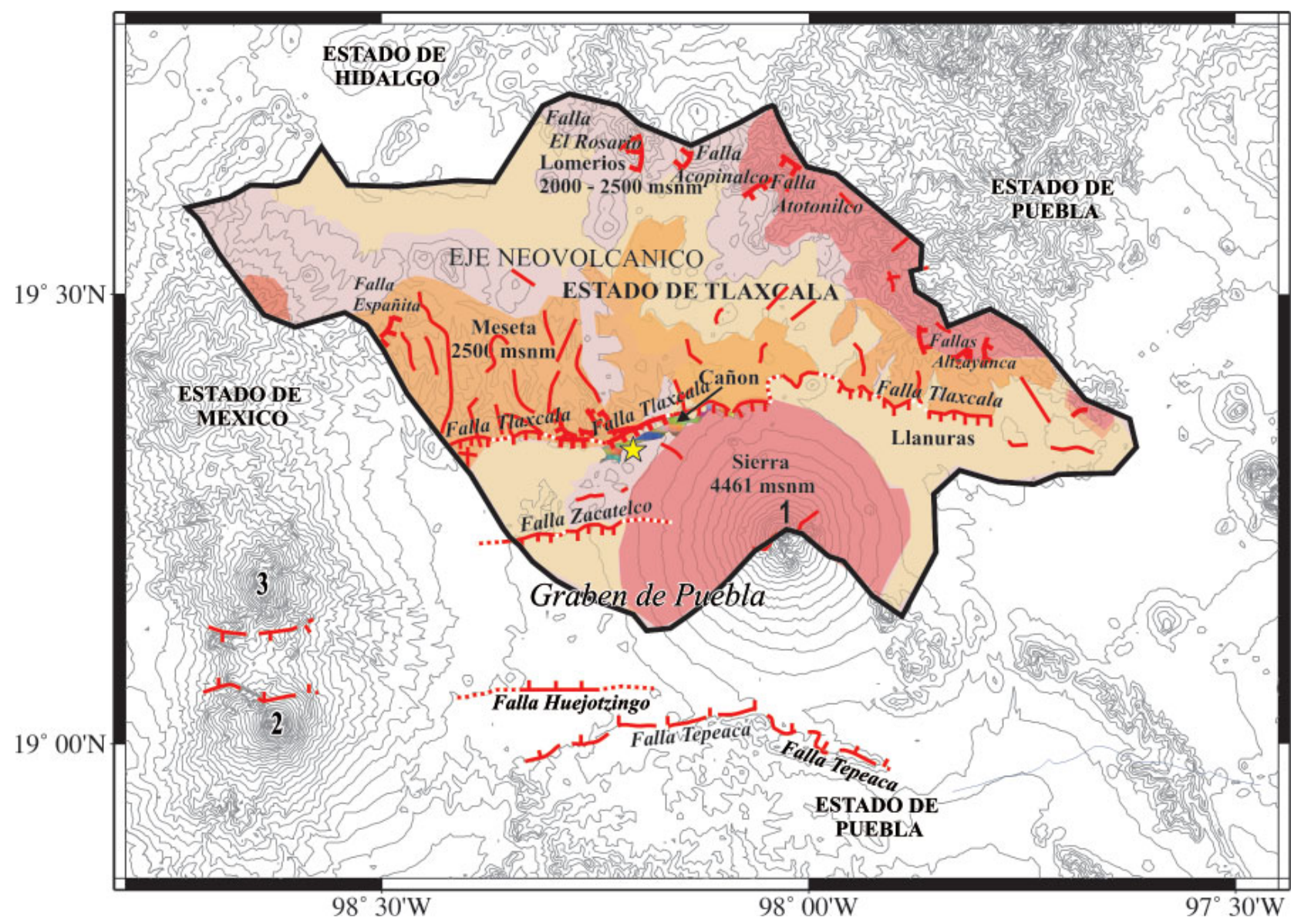

Figura 1. Mapa geomorfológico y tectónico para el estado de Tlaxcala. La estrella indica la ubicación de la ciudad de Tlaxcala (capital de este estado) y los números 1, 2 y 3 la ubicación de los volcanes: La Malinche, Popocatépetl y Iztaccihuatl, respectivamente. Las líneas rojas representan a fallas y lineamientos.

norte, formando el Graben de Puebla (Mooser et al., 1996; Santoyo et al., 2005 y García-Palomo, comunicación personal). Otras fallas menores en longitud son El Rosario, Acopinalco, Atotonilco, Altzayanca y Españita, todas con diversas orientaciones y localizadas en mayor número en el extremo Norte de este estado.

A fin de correlacionar la abrupta topografía del estado de Tlaxcala con la sismicidad local, en este estudio se ha establecido una base de datos de 45 sismos, todos con magnitudes menores a 4.0. Las características de esta sismicidad son analizadas en detalle a fin de proponer una Zonificación Sísmica para el estado de Tlaxcala que sirva como herramienta para la evaluación del riesgo y prevención de daños que pudieran ocurrir debido a la generación de sismos futuros.

\section{Sismicidad regional}

En general, los sismos que han afectado al estado de Tlaxcala han tenido su origen en tres fuentes sismogénicas. La primera y más importante fuente es el proceso de subducción de la placa de Cocos bajo la Norteamericana, misma que da origen a los sismos de gran magnitud ( $\mathrm{M}>7.0$ ) ocurridos en toda la Republica Mexicana (Suárez y Singh, 1986; Pardo y Suárez, 1995). Los eventos originados por esta fuente han producido en Tlaxcala intensidades del orden de V-VI (MM) causando alarma entre los pobladores además de daños leves en viviendas. El sismo ocurrido el 19 de Septiembre de $1985(\mathrm{Mw}=8.1)$, localizado en la costa de Michoacán a más de $400 \mathrm{~km}$ de Tlaxcala con un mecanismo que indica deformación compresional, es ejemplo de la fuente descrita anteriormente.

La segunda fuente sismogénica la constituye la deformación interna de la placa subducida, esta fuente produce sismos de menor magnitud $(\mathrm{Mw}=7.0)$ y frecuencia en comparación con la primera. Ejemplos de esta segunda fuente son los sismos del 3 de Octubre de 1864, 28 de Agosto de 1983, 24 de Octubre de 1980 y 15 de Junio de 1999, mismos que produjeron intensidades máximas de VI-VII (MM) en el área de estudio. El mecanismo focal de estos sismos obedece a una deformación de tipo extensional (Gutiérrez, 1999; Singh et al., 1999). 
La tercera fuente la constituye la deformación cortical debida a sistemas de fallas presentes en el estado. Esta fuente daría origen a sismos de magnitudes moderadas a pequeñas que producirían daños locales.

En general, la información disponible sobre la ocurrencia de sismos en el estado de Tlaxcala es escasa debido la falta de instrumentación en dicho estado. Es por ello que hasta la fecha no se conoce el potencial sísmico real del estado de Tlaxcala.

\section{Sismicidad local}

Para el estudio de la sismicidad local del estado de Tlaxcala se ha compilado y organizado una base de datos con sismos producidos entre 1984 - 2004. Estos datos provienen de las redes sísmicas del Sistema de Información Sismotelemétrica de México (SISMEX), Servicio Sismológico Nacional (SSN) y de la Red Sísmica del Valle de México (RSVM), teniendo la primera un mayor número de estaciones sísmicas y es la que ofrece una mejor cobertura azimutal sobre el estado de Tlaxcala, como se observa en la Figura 2. En la red SISMEX se registraron un total de 62 sismos, de los cuales 9 presentan formas de ondas en formato digital.

Para el procesamiento de los 62 sismos, se ha considerado (1) el programa SEISAN (Havskov y Ottemoller, 2003) para la localización hipocentral de los mismos, (2) el modelo de velocidad propuesto por Lermo et al. (2001) para la parte central de la FVM, (3) una relación de velocidades $(\mathrm{Vp} / \mathrm{Vs}=1.73)$ determinada en este estudio a partir de 120 lecturas de tiempos de llegada y (4) para estimar el tamaño de los sismos se hace uso de la relación $\mathrm{Md}^{*}=-$ $1.61947+0.00052 \mathrm{D}+2.336177 \log 10(\mathrm{~T})$, propuesta por Chavacán et. al. (2004), realizada para la parte central del FVM, que considera la duración del registro del sismo.

Una vez obtenidos los parámetros hipocentrales para el total de sismos, se seleccionan aquellos que tienen errores menores en sus parámetros hipocentrales (RMS menor o igual a 1.0 seg.) y por lo menos una lectura obtenida en la estación IIS (Figura 2).

Preliminarmente, la base de datos quedó constituida por 33 sismos locales. A estos se les añadió los parámetros hipocentrales de otros 11 sismos de la base de datos de

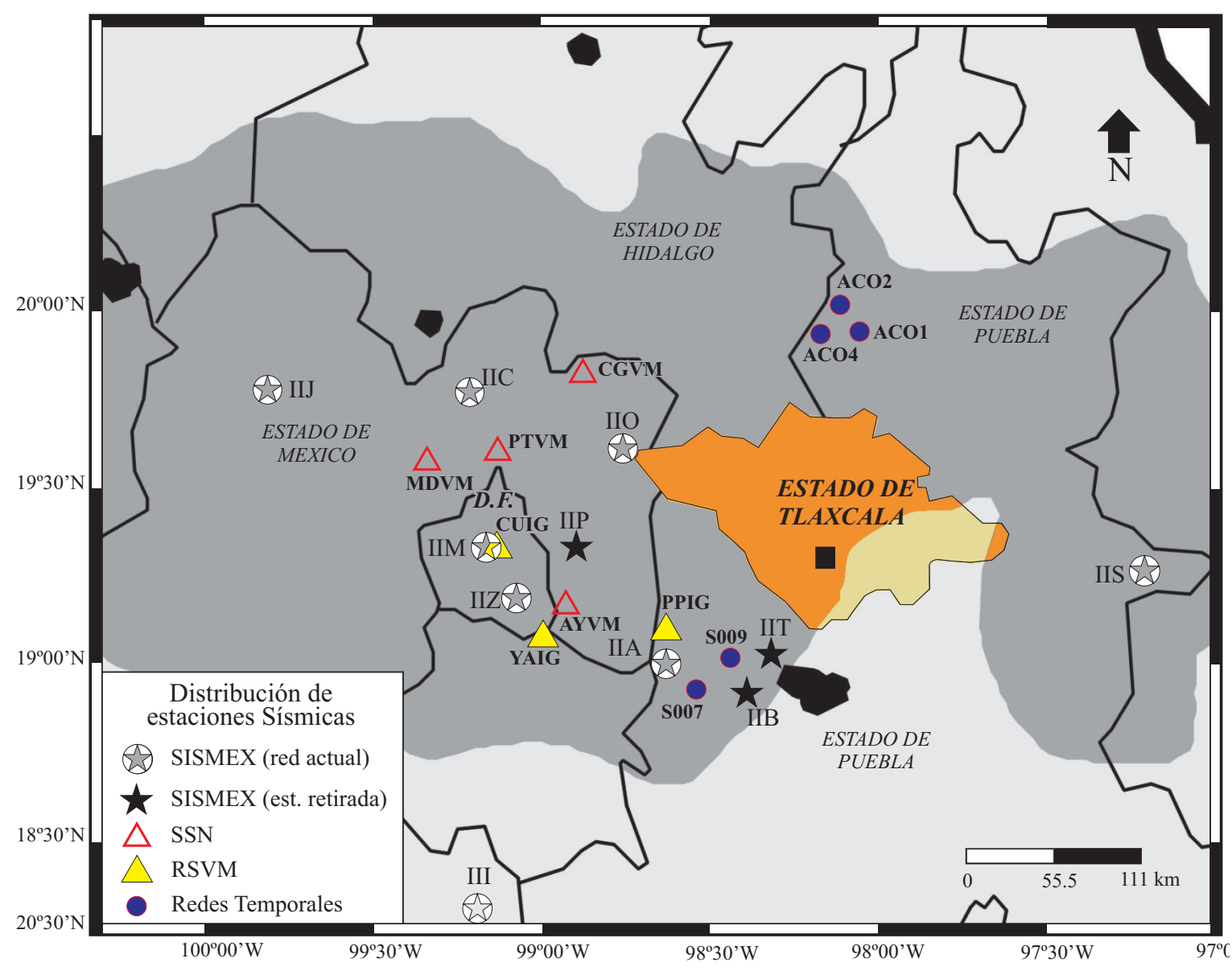

Figura 2. Distribución espacial de las estaciones sismológicas de las redes SISMEX (Sistema de Información Sismotelemétrica de México), RSVM (Red Sísmica del Valle de México) y SSN (Servicio Sismológico Nacional). La zona sombreada delimita la FVTM. 
González-Pomposo y Valdés-González (1995) provenientes de la Red Sísmica local que operó en el Estado de Puebla (RESEP) durante el periodo 1986-1989, compuesta por siete estaciones sismológicas, que junto con las estaciones de SISMEX y SSN, lograron obtener errores hypocentrales menores a los 2 seg.

Finalmente, la base de datos esta compuesta de 45 sismos ocurridos en el estado de Tlaxcala entre los años 1984 a 2004 y sus parámetros hipocentrales se muestran en la Tabla 1. De acuerdo a esta base de datos, casi el $40 \%$ de sismos se produjeron entre los meses de Septiembre y Noviembre de 1984 con magnitudes que fluctúan entre 1.6 a 2.8 Md. Para 9 eventos locales, ocurridos entre los años 1994 y 2004, se ha calculado la magnitud Mw a partir de los espectros de la amplitud de desplazamiento de la onda P.

\subsection{Análisis de la sismicidad local}

En la Figura 3, se presenta la distribución epicentral y en profundidad de los sismos considerados en este estudio. En esta figura se observa que los sismos se distribuyen de manera irregular en todo el estado de Tlaxcala; sin embargo, es posible identificar la presencia de 5 grupos de sismos con diferentes características (grupos de la A a la E).

Los sismos del Grupo A ocurrieron únicamente entre Septiembre y Diciembre de 1984; por lo que pueden ser considerados como un enjambre sísmico asociado posiblemente al sistema de la Falla Tlaxcala. Usando 46 lecturas de polaridad para la onda $\mathrm{P}$ se elaboró un mecanismo focal compuesto para este enjambre. El resultado muestra un mecanismos de falla tipo normal con una pequeña componente de desgarre siniestral y planos nodales orientados en dirección NE-SO, buzando ambos con ángulos de $45^{\circ}$ en dirección NO y SE respectivamente. Se considera que este último es el plano de falla más adecuado teniendo en cuenta los sistemas de falla predominantes en la zona, el mismo que tiene un azimut de $76^{\circ}$ con un corrimiento de $-46.1^{\circ}$.

El Grupo B se localiza al Oeste del estado de Tlaxcala. Esta actividad sísmica también podría asociarse al sistema de Falla Tlaxcala. El mecanismo focal compuesto corresponde a una falla normal con planos nodales orientados en dirección NE-SO y buzamientos de $52^{\circ}$ y $86^{\circ}$, considerando a este último el plano de falla más adecuado. El azimut de dicho plano de falla es de $80^{\circ}$ con un corrimiento de $-42.2^{\circ}$.

El Grupo C considera todos los sismos distribuídos de manera irregular en los alrededores del volcán La Malinche, estando el mayor número de sismos localizados sobre la Falla Tlaxcala y la Falla Zacatelco. Para este grupo se elaboraron mecanismos focales compuestos (sismos \# 31, 32, 33 y 35 ) y uno simple (sismo \#39), ambos corresponden a fallas normales con planos orientados en dirección NNESSO y E-O respectivamente, siendo sus buzamientos del orden de $62^{\circ}$ en dirección ESE y de $47^{\circ}$ en dirección S.
El Grupo D se ubica en el extremo Norte del estado de Tlaxcala con una aparente alineación de los sismos en dirección NE-SO; sin embargo, no se observa relación con algún elemento tectónico local que tenga una manifestación en superficie. Para este grupo no se pudo realizar su mecanismo focal por carecer de las polaridades de las estaciones que registraron los sismos, ya que solo se tomaron las localizaciones del trabajo de González-Pomposo y Valdés-González (1995).

Por último, los sismos del Grupo E se distribuyen de manera dispersa en el extremo SE del estado de Tlaxcala. El mecanismo focal simple para este grupo corresponde a una falla normal con planos orientados en dirección NNE-SSO y buzamientos del orden de $40^{\circ}$, probablemente asociado con el extremo sur del Graben de Puebla.

En la parte inferior de la Figura 3 se muestra el perfil A-A' de sismicidad orientado en dirección N-S. En este perfil se observa que los sismos se distribuyen de manera irregular alcanzando profundidades de hasta $40 \mathrm{~km}$. En general, es posible identificar la presencia de dos agrupamientos de sismos: (1) se localizan en el extremo inferior izquierdo del perfil a profundiades que oscilan entre los 28 y $40 \mathrm{~km}$. (delimitados por un círculo de línea discontinua), estos eventos se asocian, probablemente, a la deformación interna de la placa; (2) representan el conjunto con mayor número de eventos, se concentran a profundidades menores de los $10 \mathrm{~km}$, son asociados al Graben de Puebla.

De modo general en este segundo agrupamiento se observa que conforme los sismos tienden hacia el Sur del perfil aumentan en profundidad, siguiendo lineamiento con un ángulo de aproximadamente $45^{\circ}$ (indicado con una línea discontinua).

Estas observaciones sugieren que en el estado de Tlaxcala, los proceso de deformación cortical son debidos a la presencia de esfuerzos extensionales con ejes principales orientados en dirección NO-SE y E-O, lo cual coincide con lo reportado en los trabajos de Luhr et al. (1985), Suter (1991), Mooser et al. (1996), García-Palomo et al. (2002) y Santoyo et al. (2005). Asimismo, estos resultados pondrían en evidencia que la región centro y sur del estado de Tlaxcala está dominada por el Graben de Puebla cuyo rumbo es E-O.

\section{Zonificación del Estado de Tlaxcala}

De acuerdo a las características de la sismicidad local y geo-tectónicas presentes en el estado de Tlaxcala, es posible proponer un mapa de Zonificación Sísmica para dicho estado. El general, el estado de Tlaxcala puede ser dividido en zonas de alta, media y baja sismisidad, tal como se observa en la Figura 4. La zona I, de alta sismicidad se ubicaría en la región centro y sur del estado, esta actividad sísmica se asociada a la presencia de importantes fallas con orientaciones NE-SO y E-O las cuales conforman el extremo norte del Graben de Puebla. En esta zona se encuentran 
Tabla 1. Base de datos para el periodo 1984-2004

\begin{tabular}{|c|c|c|c|c|c|c|c|c|c|}
\hline $\mathbf{N}^{\circ}$ & $\begin{array}{c}\text { Fecha } \\
\text { año/mes/día }\end{array}$ & $\begin{array}{c}\text { Hora GMT } \\
\text { hora:min:seg }\end{array}$ & $\begin{array}{c}\text { Latitud } \\
\text { grados }\end{array}$ & $\begin{array}{l}\text { Longitud } \\
\text { grados }\end{array}$ & $\begin{array}{c}\text { Prof. } \\
\text { km }\end{array}$ & $\begin{array}{c}\text { RMS } \\
\text { seg }\end{array}$ & $\begin{array}{l}\text { Mag } \\
\text { Md }\end{array}$ & $\begin{array}{l}\text { iitud } \\
\text { Mw }\end{array}$ & Fuente \\
\hline 1 & $1984 / 9 / 25$ & $10: 3: 59.8$ & 19.405 & -98.021 & 12.2 & 0.7 & 1.9 & & SISMEX \\
\hline 2 & $1984 / 9 / 25$ & $10: 4: 56.7$ & 19.440 & -97.973 & 17.6 & 1 & 1.8 & & SISMEX \\
\hline 3 & $1984 / 9 / 26$ & $17: 5: 46.5$ & 19.475 & -98.108 & 6 & 0.7 & 2.5 & & SISMEX \\
\hline 4 & $1984 / 9 / 29$ & $17: 8: 6.9$ & 19.480 & -98.149 & 6.7 & 0.4 & 2.3 & & SISMEX \\
\hline 5 & $1984 / 9 / 29$ & $17: 35: 13.9$ & 19.512 & -98.091 & 4.9 & 0.9 & 2.4 & & SISMEX \\
\hline 6 & $1984 / 9 / 30$ & $0: 23: 35.3$ & 19.478 & -98.185 & 7.3 & 0.1 & 2.6 & & SISMEX \\
\hline 7 & $1984 / 9 / 30$ & $4: 9: 56.6$ & 19.468 & -98.193 & 7.7 & 0.1 & 2.6 & & SISMEX \\
\hline 8 & $1984 / 9 / 30$ & $14: 51: 55.2$ & 19.489 & -98.081 & 4.8 & 1 & 2.7 & & SISMEX \\
\hline 9 & $1984 / 10 / 1$ & $4: 35: 1.4$ & 19.153 & -98.189 & 26.6 & 0.1 & 1.9 & & SISMEX \\
\hline 10 & $1984 / 10 / 2$ & $0: 20: 42.3$ & 19.438 & -98.159 & 6.9 & 0.7 & 2.4 & & SISMEX \\
\hline 11 & $1984 / 10 / 2$ & $3: 59: 39.9$ & 19.325 & -98.100 & 13.7 & 0.1 & 1.6 & & SISMEX \\
\hline 12 & $1984 / 10 / 2$ & $15: 16: 54$ & 19.464 & -98.200 & 7.7 & 0.1 & 2.8 & & SISMEX \\
\hline 13 & $1984 / 10 / 2$ & $16: 40: 20.5$ & 19.476 & -98.171 & 7 & 0.3 & 2.8 & & SISMEX \\
\hline 14 & $1984 / 10 / 2$ & $19: 44: 21.3$ & 19.479 & -98.208 & 0.7 & 1 & 2.1 & & SISMEX \\
\hline 15 & $1984 / 10 / 2$ & $20: 10: 57.3$ & 19.460 & -98.165 & 7 & 0.9 & 2.1 & & SISMEX \\
\hline 16 & $1984 / 10 / 2$ & $22: 1: 56$ & 19.473 & -98.133 & 6.8 & 0.3 & 2.1 & & SISMEX \\
\hline 17 & $1984 / 10 / 3$ & $1: 11: 18.4$ & 19.517 & -98.105 & 5.4 & 0.8 & 2.6 & & SISMEX \\
\hline 18 & $1984 / 10 / 3$ & $1: 19: 34.4$ & 19.488 & -98.183 & 7.4 & 0.6 & 2.2 & & SISMEX \\
\hline 19 & 1984 / $11 / 6$ & $1: 55: 57.8$ & 19.408 & -98.266 & 5.7 & 0.4 & 2.0 & & SISMEX \\
\hline 20 & $1986 / 3 / 20$ & $10: 12: 34$ & 19.340 & -97.610 & 20 & 1.7 & 3.4 & & RESEP \\
\hline 21 & $1986 / 5 / 8$ & $18: 44: 30.2$ & 19.668 & -98.667 & 30 & 1.9 & 4.0 & & RESEP \\
\hline 22 & $1986 / 5 / 11$ & $12: 27: 26.2$ & 19.657 & -98.650 & 29 & 2 & 4.1 & & RESEP \\
\hline 23 & $1986 / 8 / 30$ & $19: 23: 46.6$ & 19.680 & -98.571 & 37 & 0.4 & 4.0 & & RESEP \\
\hline 24 & $1986 / 8 / 30$ & $22: 34: 23.5$ & 19.691 & -98.613 & 37 & 0.5 & 3.4 & & RESEP \\
\hline 25 & $1986 / 9 / 1$ & $3: 40: 33.5$ & 19.636 & -98.687 & 37 & 1.9 & 3.4 & & RESEP \\
\hline 26 & $1987 / 2 / 18$ & $1: 26: 37$ & 19.240 & -97.620 & 24 & 0.7 & 3.1 & & RESEP \\
\hline 27 & $1987 / 4 / 18$ & $8: 30: 42$ & 19.530 & -98.330 & 37 & 0.7 & 3.1 & & RESEP \\
\hline 28 & $1987 / 8 / 16$ & $9: 3: 54$ & 19.390 & -98.170 & 32 & 1 & 3.1 & & RESEP \\
\hline 29 & $1988 / 2 / 1$ & $7: 44: 56$ & 19.250 & -97.580 & 28 & 1.6 & 2.0 & & RESEP \\
\hline 30 & $1988 / 7 / 25$ & $4: 31: 7.5$ & 19.386 & -98.167 & 24 & 0.9 & 2.7 & & SISMEX \\
\hline 31 & $1988 / 12 / 29$ & $5: 24: 33.6$ & 19.320 & -98.204 & 3.1 & 0.4 & 2.1 & & SISMEX \\
\hline 32 & $1988 / 12 / 29$ & $5: 25: 15.8$ & 19.266 & -98.256 & 4.7 & 0.3 & 3.0 & & SISMEX \\
\hline 33 & $1988 / 12 / 29$ & $5: 49: 33.7$ & 19.318 & -98.208 & 3 & 0.3 & 2.3 & & SISMEX \\
\hline 34 & $1989 / 7 / 8$ & $6: 27: 45$ & 19.330 & -97.690 & 32 & 1.2 & 3.0 & & RESEP \\
\hline 35 & $1989 / 8 / 5$ & $23: 42: 16.1$ & 19.269 & -98.216 & 8.5 & 0.3 & 3.0 & & SISMEX \\
\hline 36 & $1994 / 5 / 17$ & $11: 21: 27.6$ & 19.359 & -98.416 & 4.4 & 0.1 & 2.2 & 2.2 & SISMEX \\
\hline 37 & $1995 / 1 / 5$ & $20: 15: 49.9$ & 19.362 & -98.445 & 8.6 & 0.2 & 2.1 & 2.1 & SISMEX \\
\hline 38 & $1995 / 4 / 9$ & $15: 55: 39.1$ & 19.360 & -98.418 & 10 & 0.1 & 2.2 & 2.2 & SISMEX \\
\hline 39 & $1996 / 9 / 6$ & $6: 55: 0$ & 19.148 & -98.319 & 6.6 & 0.3 & 3.0 & 3.0 & SISMEX \\
\hline 40 & $1997 / 4 / 23$ & $12: 46: 23.3$ & 19.155 & -98.170 & 4.2 & 0.1 & 2.4 & 2.3 & SISMEX \\
\hline 41 & $1997 / 5$ / 6 & $8: 0: 5.9$ & 19.146 & -98.184 & 3.1 & 0.3 & 2.7 & 2.7 & SISMEX \\
\hline 42 & $1997 / 6 / 18$ & $21: 12: 2.2$ & 19.327 & -98.429 & 4.4 & 0.1 & 2.3 & 2.3 & SISMEX \\
\hline 43 & $1997 / 6 / 18$ & $21: 15: 6.2$ & 19.336 & -98.422 & 5.8 & 0.1 & 2.4 & 2.4 & SISMEX \\
\hline 44 & $2002 / 11 / 3$ & $21: 35: 0.4$ & 19.273 & -98.219 & 1 & 1.3 & 3.5 & & RSVM \\
\hline 45 & $2004 / 8 / 31$ & $7: 54: 5.3$ & 19.232 & -97.681 & 5.2 & 0.5 & 2.9 & 3.0 & SISMEX \\
\hline
\end{tabular}

SISMEX: Sistema de Información Sismotelemétrica de México, RESEP: Red Sísmica del Estado de Puebla y RSVM: Red Sísmica del Valle de México. 

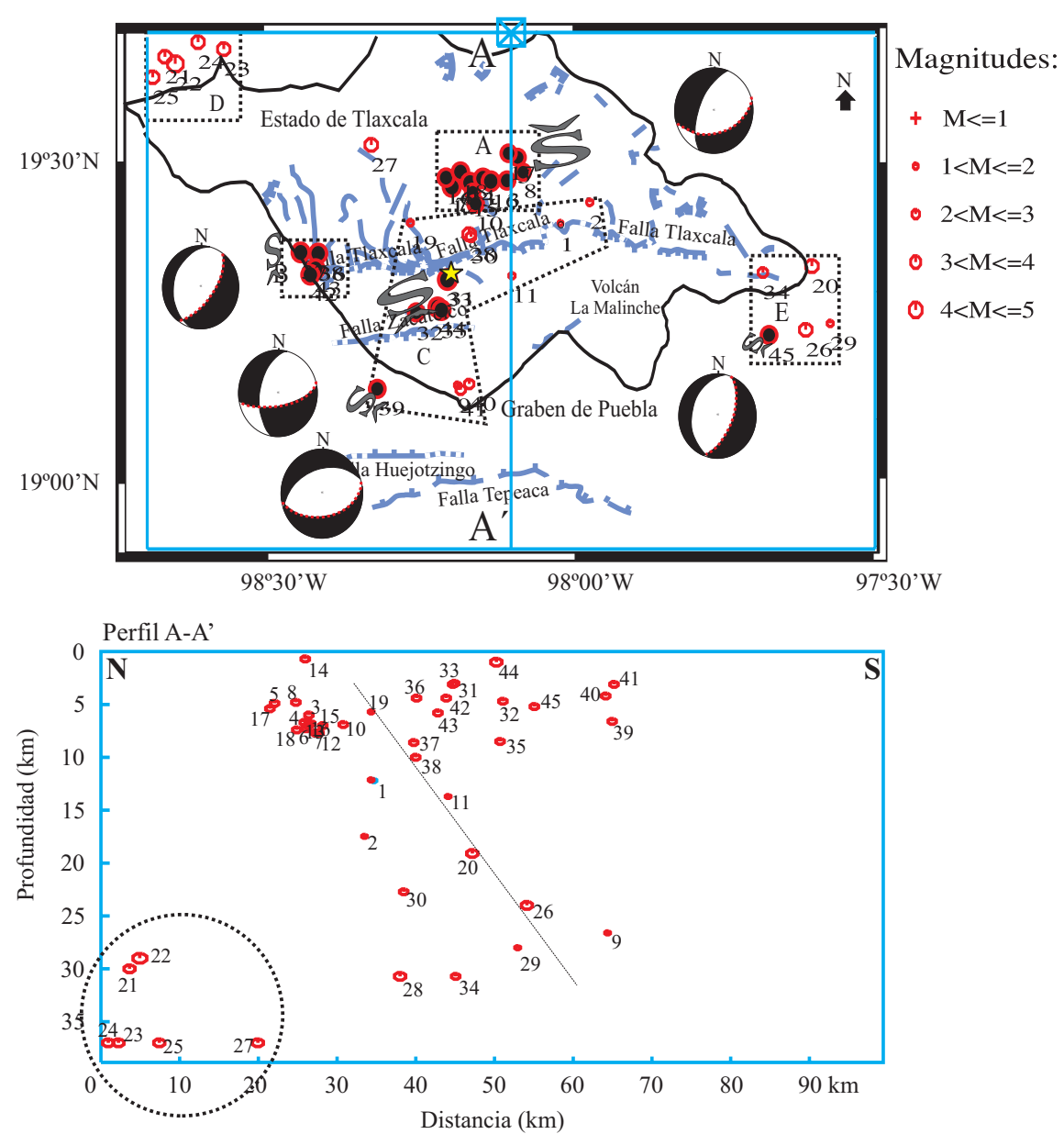

Figura 3. Parte superior: Distribución espacial de la sismicidad (45 eventos). Los recuadros de líneas discontinuas indican los diferentes agrupamientos de esta sismicidad. La estrella indica la localización de la capital de este estado, las líneas continuas indican lineamientos y fallas. Los sismos de color negro, fueron utilizados para los mecanismos focales simples y compuestos. Parte inferior: Perfíl A-A', muestra la distribución en profundidad de los 45 sismos. El círculo localizado en el extremo inferior izquierdo del perfil, delimita los eventos localizados en profundiades que oscilan entre los 28 y $40 \mathrm{~km}$ y que pueden asociarse a la deformación interna de la placa.

las ciudades más importantes por su densidad poblacional como son: Tlaxcala (capital del estado) Chiautempan, Huamantla y Apizaco. La zona II, de sismicidad media se localiza al NO del estado y abarca un área pequeña en la cual no ha sido posible identificar algunos elementos tectónicos superficiales. La zona III, de baja sismicidad considera las áreas en las cuales no se habrían producido sismos. Así mismo, tampoco se ha identificado la existencia de elementos tectónicos que sugieran la presencia de deformación actual.

El mapa de Zonificación Sísmica propuesta es preliminar debido a que la información sísmica utilizada en este estudio no es representativa del potencial sísmico del estado de Tlaxcala.

\section{Conclusiones}

La relación entre la distribución espacial de la sismicidad local ocurrida en estado de Tlaxcala (1984-
2004) y la geotectónica del mismo ha permitido proponer un mapa de Zonificación Sísmica para este estado. Según esta zonificación, la Zona I, que abarca la región centro y sur del estado, considera el área en donde se tiene la mayor ocurrencia de sismos y la presencia de varios sistemas de fallas, probablemente asociados al extremo norte del Graben de Puebla.

El mapa de Zonificación Sísmica para el estado de Tlaxcala, sugiere la necesidad de instalar una red sísmica local que permita cuantificar de mejor manera la deformación local que se produce en todo el estado. Asimismo, es importante ampliar y mejorar los estudios geofísicos en el estado de Tlaxcala a fin de entender mejor el comportamiento del Graben de Puebla.

\section{Agradecimientos}

Se agradece al Dr. García-Palomo, por la información proporcionada sobre la tectónica de la zona de estudio. 


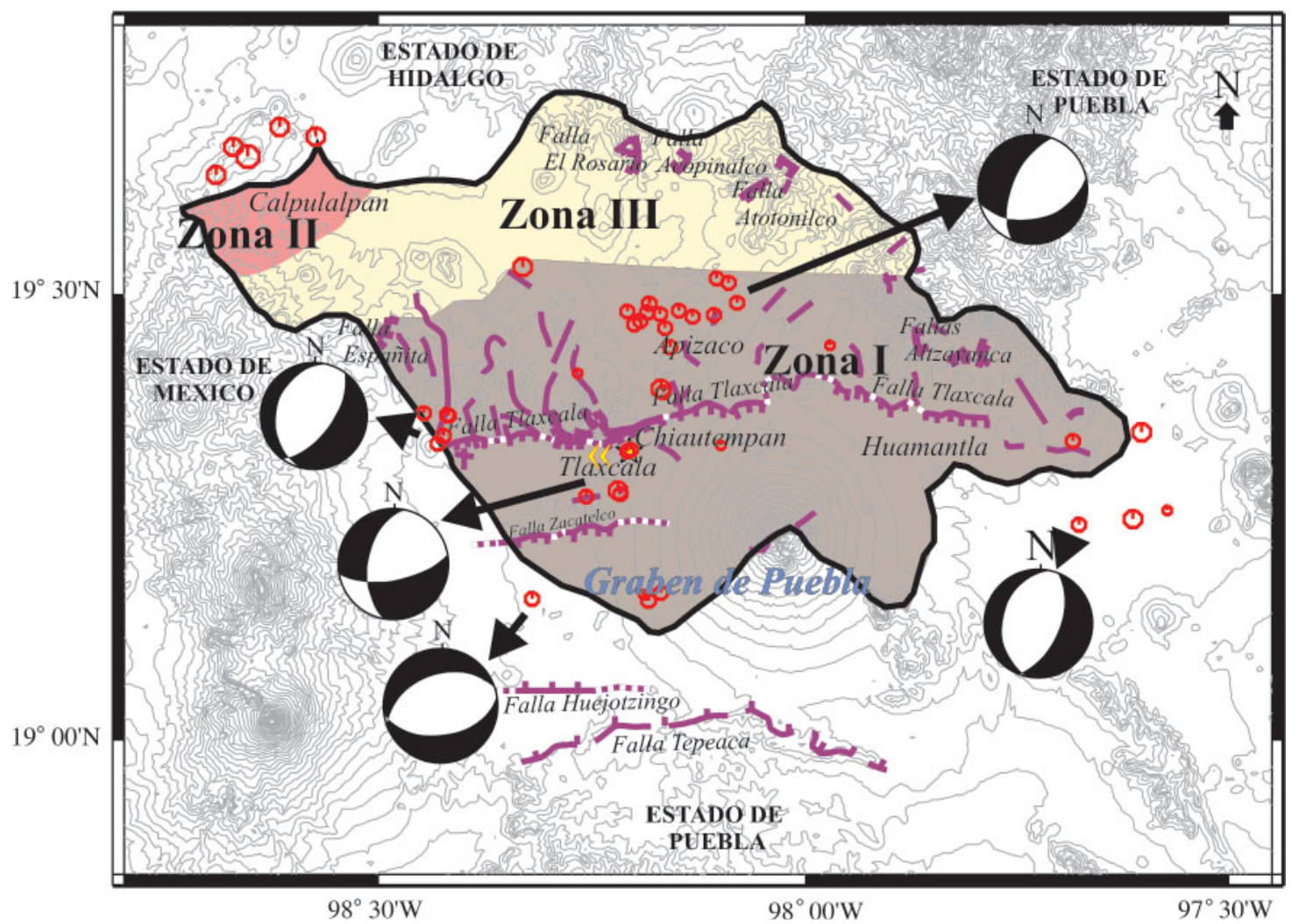

Figura 4. Mapa de Zonificación Sísmica para el estado de Tlaxcala en función de la distribución de los sismos y las características sismotectónicas locales. La estrella indica la ubicación de la capital del estado.

Al Dr. Quintanar, por los datos sísmicos tomados de los catálogos de SSN y RSVM, así como por sus comentarios y observaciones. Al instituto de Ingeniería de la UNAM por todas las facilidades otorgadas durante la elaboración de este trabajo.

\section{Referencias bibliográficas}

Chavacán, M., Lermo, J., Quintanar, L., 2004, Determinación de una escala de magnitud para temblores corticales en la parte central del Cinturón Volcánico Mexicano, en I Conf. Int. Peligrosidad e Ingeniería Sísmica, Universidad de Oriente, Santiago de Cuba, Cuba, CD.ROM.

Figueroa, J., 1974, Sismicidad en Puebla, Macrosismo del 28 de agosto de 1973: México, D.F., Instituto de Ingeniería, UNAM, 343, 64p.

Gutiérrez, C., 1999, Mapa de intensidades sísmicas del evento del 15 de junio de 1999, Mw 7.0, Tehuacan Puebla, en XII Congreso Nacional de Ingeniería Sísmica: Morelia, Mich., México, Sociedad Mexicana de Ingeniería Sísmica, 736-743.

González-Pomposo, G. J. y Valdés-González, C., 1995, Seismicity of the state of Puebla, México 1986-1989: Geofísica Internacional, 34, 221-232.

García-Palomo, A., Macías, J.L., Tolson, G., Valdez, G., Mora, J.C., 2002, Volcanic stratigraphy and Geological evolution of the Apan Region, east-central sector of the Trans-Mexican Volcanic Belt: Geofísica Internacional, 41-2, 133-150.

Havskov, J., Ottemoller, L., 2003, SEISAN: The earthquake analysis software, version 8.0: Bergen, Norway, Department of Earth Science, University of Bergen (ed.), $250 \mathrm{p}$.

Lermo, J., Havskov, J., Soto, J., 2001, Sistema de información sismotelemétrica de México (SISMEX). Veintisiete años de servicio, en XIII Congreso Nacional de Ingeniería Sísmica: Guadalajara, Jal. México, Sociedad Mexicana de Ingeniería Sísmica, artículo T11A06, $17 \mathrm{p}$.

Luhr, A., Nelson, J., Allan, F., Carmichael, I.S.E., 1985, Active rifting in southwestern México: manifestation of an incipient eastward spreading-ridge jump: Geology, 13, 54-57.

Mooser, F., Montiel, A., Zúñiga, Á., 1996, Nuevo mapa geológico de las cuencas de México, Toluca y Puebla: México, D.F., Subdirección de Construcción, Comisión Federal de Electricidad México, D.F., $63 p$.

Pardo, M., Suárez, G., 1995, Shape of the subducted Rivera and Cocos plates in southern México: Seismic and tectonic implication: Journal of Geophysical Research, 100, 12357-12373.

Santoyo, E., Ovando, E., Mooser, F., León, E., 2005, Síntesis geotécnica de la cuenca del Valle de México: México, D.F., TGC geotecnia S.A. de C.V., 171p.

Singh, S.K., Ordaz, M., Pacheco, J.F., Quaas, R., Alcántara, L., Alcocer, S., Gutiérrez, C., Meli, R., Ovando, E., 1999, A preliminary report on the Tehuacán, México earthquake of June 15, 1999 (Mw=7.0): Seismological Research Letters, 70-5, 489-504.

Suárez, G., Singh, S.K., 1986, Tectonic interpretation of the TransMexican Volcanic Belt-Discussion: Tectonophys, 127, 155-160.

Suter, M., 1991, State of stress and active deformation in México and Central América, in Slemmons, D.B., et al., Eds., Neotectonics of North América: Geological Society of America, decade of North America geology, decade Map., 1, 401-421.

Manuscrito recibido: Diciembre 5, 2006

Manuscrito corregido recibido: Febrero 6, 2007

Manuscrito aceptado: Febrero 15, 2007 